\title{
A NEW VARIATIONAL FORMULATION FOR CONVEX HAMILTONIAN SYSTEMS WITH NONLINEAR BOUNDARY CONDITIONS
}

\author{
MARK LEWIS and ABBAS MOAMENI ${ }^{凶}$
}

(Received 1 April 2010)

\begin{abstract}
A variational principle is established to provide a new formulation for convex Hamiltonian systems. Using this formulation, we obtain some existence results for second-order Hamiltonian systems with a variety of boundary conditions, including nonlinear ones.
\end{abstract}

2010 Mathematics subject classification: primary 37K05; secondary 65K10.

Keywords and phrases: convex Hamiltonian systems, variational principles, variational methods, duality theory.

\section{Introduction}

This paper is a continuation of [4-7] where a general nonlinear selfdual variational principle was established to provide a variational formulation and resolution for several nonlinear partial differential equations. Applications included nonlinear transport equations, Navier-Stokes equations, and the generalized Choquard-Pekar Schrödinger equations with certain nonlocal potentials. In this paper we introduce a new variational formulation for second-order convex Hamiltonian systems to construct solutions that satisfy certain linear and nonlinear boundary conditions. To illustrate this principle we consider a second-order Hamiltonian system with periodic boundary conditions of the form

$$
\left\{\begin{array}{l}
A(t) u(t)-\ddot{u}(t)=\nabla \Phi(t, u(t)), \\
u(0)=u(T), \dot{u}(0)=\dot{u}(T),
\end{array}\right.
$$

and also with nonlinear boundary conditions,

$$
\left\{\begin{array}{l}
A(t) u(t)-\ddot{u}(t)=\nabla \Phi(t, u(t)), \\
\dot{u}(0)=-\nabla \Psi_{1}(u(0)), \\
\dot{u}(T)=\nabla \Psi_{2}(u(T)) .
\end{array}\right.
$$

In the above systems the Hamiltonian $\Phi:[0, T] \times \mathbb{R}^{N} \rightarrow \mathbb{R} \cup\{\infty\}$ is convex and lower semi-continuous on the second variable. Also the functions $\Psi_{1}, \Psi_{2}: \mathbb{R}^{N} \rightarrow \mathbb{R} \cup\{\infty\}$

(C) 2011 Australian Mathematical Publishing Association Inc. 0004-9727/2011 \$16.00 
are convex and lower semi-continuous and the matrix-valued map $A \in C([0, T]$, $\mathbb{R}^{N} \times \mathbb{R}^{N}$ ) is symmetric. Letting $\langle\cdot, \cdot\rangle$ denote the usual inner product on $\mathbb{R}^{N}$, the Fenchel-Legendre dual of $\Phi(t, \cdot)$ is denoted by $\Phi^{*}(t, \cdot)$ and defined by

$$
\Phi^{*}(t, v)=\sup _{u \in \mathbb{R}^{N}}\{\langle v, u\rangle-\Phi(t, u)\}
$$

We now state our first theorem.

Theorem 1.1. Suppose that $\Phi:[0, T] \times \mathbb{R}^{N} \rightarrow \mathbb{R}$ is measurable with respect to the $\sigma$-field generated by the product of Lebesgue sets in $[0, T]$ and Borel sets in $\mathbb{R}^{N}$ and is convex and lower semi-continuous in the second variable. Let $A \in C([0, T]$, $\mathbb{R}^{N} \times \mathbb{R}^{N}$ ) be a strictly positive definite symmetric matrix-valued map. If $\Phi$ is Gâteaux differentiable with respect to the second variable, then critical points of the functional

$$
I(u)=\int_{0}^{T} \Phi^{*}(t, A(t) u(t)-\ddot{u}(t)) d t-\int_{0}^{T} \Phi(t, u(t)) d t
$$

on the space of periodic paths are solutions of the system (1.1) and vice versa.

Theorem 1.1 applies readily to many equations, giving a new formulation and resolution. In the following example we shall show how the new functional $I$ given by (1.3) will be useful in the calculus of variations to obtain solutions with more regularity. Indeed, we consider the system

$$
\left\{\begin{array}{l}
A(t) u(t)-\ddot{u}(t)=|u(t)|^{p-2} u(t)+f(t), \\
u(0)=u(T), \dot{u}(0)=\dot{u}(T)
\end{array}\right.
$$

As a consequence of Theorem 1.1 we shall establish the following existence result.

Theorem 1.2. Let $A \in C\left([0, T], \mathbb{R}^{N} \times \mathbb{R}^{N}\right)$ be a strictly positive definite symmetric matrix-valued map. Let $p>2$ and $f \in L^{p^{\prime}}[0, T]$ where $p^{\prime}=p /(p-1)$. If $\|f\|_{L^{p^{\prime}}[0, T]}$ is small enough, then the functional

$$
\begin{aligned}
I(u)=\frac{1}{p^{\prime}} & \int_{0}^{T}|-\ddot{u}(t)+A(t) u(t)-f(t)|^{p^{\prime}} d t-\frac{1}{p} \int_{0}^{T}|u(t)|^{p} d t \\
& -\int_{0}^{T}\langle f(t), u(t)\rangle d t
\end{aligned}
$$

has a nontrivial critical point on the space of periodic paths in $W^{2, p^{\prime}}[0, T]$ which is indeed a solution of (1.4).

Here is our result taking into account certain nonlinear boundary conditions.

Theorem 1.3. Suppose that $\Phi:[0, T] \times \mathbb{R}^{N} \rightarrow \mathbb{R}$ is measurable with respect to the $\sigma$-field generated by the product of Lebesgue sets in $[0, T]$ and Borel sets in $\mathbb{R}^{N}$ and is Gâteaux differentiable, convex and lower semi-continuous on the second variable. Let $A \in C\left([0, T], \mathbb{R}^{N} \times \mathbb{R}^{N}\right)$ be a strictly positive definite symmetric matrix-valued map. We also assume that $\Psi_{1}, \Psi_{2}: \mathbb{R}^{N} \rightarrow \mathbb{R}$ are Gâteaux differentiable, convex and lower 
semi-continuous. Then critical points of the functional

$$
\begin{aligned}
I(u)= & \int_{0}^{T} \Phi^{*}(t, A(t) u(t)-\ddot{u}(t)) d t-\int_{0}^{T} \Phi(t, u(t)) d t \\
& +\Psi_{1}^{*}(-\dot{u}(0))+\Psi_{2}^{*}(\dot{u}(T))-\Psi_{1}(u(0))-\Psi_{2}(u(T))
\end{aligned}
$$

are solutions of the system (1.2) and vice versa.

As an application of Theorem 1.3, we establish the following existence result for (1.2).

Theorem 1.4. Let $\Phi:[0, T] \times \mathbb{R}^{N} \rightarrow \mathbb{R}$ be continuously differentiable, strictly convex and lower semi-continuous in the second variable. Let $\Psi_{1}, \Psi_{2}: \mathbb{R}^{N} \rightarrow \mathbb{R}$ be continuously differentiable and strictly convex. Then the functional

$$
\begin{aligned}
I(u)= & \int_{0}^{T} \Phi^{*}(t, A(t) u(t)-\ddot{u}(t)) d t-\int_{0}^{T} \Phi(t, u(t)) d t \\
& +\Psi_{1}^{*}(-\dot{u}(0))+\Psi_{2}^{*}(\dot{u}(T))-\Psi_{1}(u(0))-\Psi_{2}(u(T))
\end{aligned}
$$

has a critical point $\widetilde{u} \in W^{2, p^{\prime}}[0, T]$ which is a solution of (1.2) if the following conditions hold for all $t \in[0, T]$ and $\xi \in \mathbb{R}^{N}$ :

$$
\begin{gathered}
\Phi(t, 0)=\Psi_{i}(0)=0 \text {, and there exist } p, p_{i}>2, i=1,2 \text {, such that } \\
\qquad \begin{aligned}
p \Phi(t, \xi) \leq\langle\nabla \Phi(t, \xi), \xi\rangle, \\
p_{i} \Psi_{i}(\xi) \leq\left\langle\nabla \Psi_{i}(\xi), \xi\right\rangle ;
\end{aligned}
\end{gathered}
$$

(ii) there exist $q \geq p$ and $\alpha>0, q_{i}>p_{i}$ and $\alpha_{i}>0, i=1,2$, such that

$$
\begin{aligned}
\Phi(t, \xi) & \leq \alpha|\xi|^{q}, \\
\Psi_{i}(\xi) & \leq \alpha_{i}|\xi|^{q_{i}} .
\end{aligned}
$$

Note that the functions $\Phi(t, \xi)=|\xi|^{p}$ and $\Psi_{i}(\xi)=|\xi|^{p_{i}}$ for $p, p_{i}>2$ satisfy conditions (i) and (ii) in the above theorem. This is a more general version of the problem considered in [1].

\section{Preliminaries}

In this section we recall some standard notions in convex analysis, Sobolev spaces and nonsmooth critical point theory $[2,3,8]$. Let $X$ be a reflexive Banach space and $X^{*}$ its topological dual. Let $\Phi: X \rightarrow \mathbb{R} \cup\{\infty\}$ be a proper convex function. Define the sub-differential $\partial \Phi$ of $\Phi$ to be the following set-valued operator: if $u \in \operatorname{Dom}(\Phi)$, set

$$
\partial \Phi(u)=\left\{p \in X^{*} ;\langle p, v-u\rangle+\Phi(u) \leq \Phi(v) \text { for all } v \in X\right\}
$$

and if $u \notin \operatorname{Dom}(\Phi)$, set $\partial \Phi(u)=\varnothing$. If $\Phi$ is Gâteaux differentiable at $u$ then $\partial \Phi(u)=$ $\{\nabla \Phi(u)\}$.

The Fenchel-Legendre dual of $\Phi$ is denoted by $\Phi^{*}$ and is the function on $X^{*}$ defined to be

$$
\Phi^{*}(p)=\sup \{\langle p, x\rangle-\Phi(x) ; x \in X\} .
$$


It follows from this definition that for every $(u, p) \in X \times X^{*}$, we have $\Phi(u)+\Phi^{*}(p) \geq$ $\langle u, p\rangle$. If $\Phi$ is convex and lower semi-continuous, then $\Phi^{* *}=\Phi$ and the following are equivalent:

(i) $\Phi(u)+\Phi^{*}(p)=\langle u, p\rangle$;

(ii) $p \in \partial \Phi(u)$;

(iii) $u \in \partial \Phi^{*}(p)$.

Let $r>1$ be a real number and $k$ be a positive integer. Let $L^{r}[0, T]$ be the classical space of integrable functions from $[0, T]$ to $\mathbb{R}^{N}$ equipped with the norm

$$
\|u\|_{L^{r}[0, T]}=\left(\int_{0}^{T}|u(t)|^{r} d t\right)^{1 / r}
$$

and $W^{k, r}[0, T]$ the classical Sobolev space consisting of all paths from $[0, T]$ to $\mathbb{R}^{N}$ such that the derivatives up to order $k$ exist in the sense of distributions and equipped with the norm

$$
\|u\|_{W^{k, r}[0, T]}=\|u\|_{L^{r}[0, T]}+\left\|\frac{d^{k} u}{d t^{k}}\right\|_{L^{r}[0, T]} .
$$

Set $W_{\text {per }}^{k, r}[0, T]$ to be the space of periodic maps in $W^{k, r}[0, T]$, that is,

$$
W_{\mathrm{per}}^{k, r}[0, T]=\left\{u \in W^{k, r}[0, T] ; \frac{d^{i} u(T)}{d t^{i}}=\frac{d^{i} u(0)}{d t^{i}}, i=1,2, \ldots, k-1\right\},
$$

equipped with the norm of $W^{k, r}[0, T]$.

Since, the functionals proposed in Theorems 1.1 and 1.3 may not be Gâteaux differentiable, we are required to give a meaning to the notion of a critical point of such a functional.

Definition 2.1. We will say that $u \in W_{\text {per }}^{2, r}[0, T]$ is a critical point of

$$
I(u)=\int_{0}^{T}\left[\Phi^{*}(t, A(t) u(t)-\ddot{u}(t))-\Phi(t, u(t))\right] d t
$$

if $I(u)$ is finite and there exist $v(t) \in \partial \Phi^{*}(t, A(t) u(t)-\ddot{u}(t))$ such that

$$
\int_{0}^{T}\langle v(t), A(t) \eta(t)\rangle d t-\int_{0}^{T}\langle v(t), \ddot{\eta}(t)\rangle d t-\int_{0}^{T}\langle\nabla \Phi(t, u(t)), \eta(t)\rangle d t=0,
$$

for all $\eta \in W_{\text {per }}^{2, r}[0, T]$.

Definition 2.2. We will say that $u \in W^{2, r}[0, T]$ is a critical point of

$$
\begin{aligned}
I(u)= & \int_{0}^{T}\left[\Phi^{*}(t, A(t) u(t)-\ddot{u}(t))-\Phi(t, u(t))\right] d t \\
& +\Psi_{1}^{*}(-\dot{u}(0))+\Psi_{2}^{*}(\dot{u}(T))-\Psi_{1}(u(0))-\Psi_{2}(u(T))
\end{aligned}
$$

if $I(u)$ is finite and there exist $v(t) \in \partial \Phi^{*}(t, A(t) u(t)-\ddot{u}(t)), \quad w_{1} \in \partial \Psi_{1}^{*}(-\dot{u}(0))$ 
and $w_{2} \in \partial \Psi_{2}^{*}(\dot{u}(T))$ such that

$$
\begin{aligned}
0= & \int_{0}^{T}\langle v(t), A(t) \eta(t)\rangle d t-\int_{0}^{T}\langle v(t), \ddot{\eta}(t)\rangle d t-\int_{0}^{T}\langle\nabla \Phi(t, u(t)), \eta(t)\rangle d t \\
& +\left\langle w_{1},-\dot{\eta}(0)\right\rangle+\left\langle w_{2}, \dot{\eta}(T)\right\rangle-\left\langle\nabla \Psi_{1}(u(0)), \eta(0)\right\rangle-\left\langle\nabla \Psi_{2}(u(T)), \eta(T)\right\rangle,
\end{aligned}
$$

for all $\eta \in W^{2, r}[0, T]$.

\section{Proofs}

In this section we proceed with the proof of theorems stated in the Introduction. We need a few preliminary results that will be frequently used in the proofs.

Suppose that the matrix $A(t)$ is strictly positive and continuous, thus there exist positive constants $\alpha_{1}$ and $\alpha_{2}$ such that

$$
\alpha_{1}^{2}|\xi|^{2} \leq\langle A(t) \xi, \xi\rangle \leq \alpha_{2}^{2}|\xi|^{2}
$$

for all $\xi \in \mathbb{R}^{N}$. Now for each $u \in W^{2, r}[0, T]$ define

$$
\|u\|=\|-\ddot{u}(t)+A(t) u(t)\|_{L^{r}[0, T]}+|\dot{u}(0)|+|\dot{u}(T)|,
$$

and for each $u \in W_{\text {per }}^{2, r}[0, T]$ define

$$
\|u\|_{\text {per }}=\|-\ddot{u}+A(t) u\|_{L^{r}[0, T]} .
$$

We have the following proposition.

Proposition 3.1.

(1) $\|u\|$ is an equivalent norm for $W^{2, r}[0, T]$.

(2) $\|u\|_{\text {per }}$ is an equivalent norm for $W_{\text {per }}^{2, r}[0, T]$.

Proof. We prove part (2); the same argument works for part (1). It follows from (3.1) that

$$
\begin{aligned}
\|u\|_{\mathrm{per}} & =\|-\ddot{u}+A(t) u\|_{L^{r}[0, T]} \\
& \leq\|-\ddot{u}\|_{L^{r}[0, T]}+\|A(t) u\|_{L^{r}[0, T]} \\
& \leq\|-\ddot{u}\|_{L^{r}[0, T]}+\alpha_{2}\|u\|_{L^{r}[0, T]} \\
& \leq\left(1+\alpha_{2}\right)\|u\|_{W^{2, r}[0, T]} .
\end{aligned}
$$

Now suppose that $\|u\|_{\text {per }}<\infty$. Set $f(t)=-\ddot{u}(t)+A(t) u(t)$ so $f \in L^{r}[0, T]$. It follows that

$$
\begin{aligned}
\int_{0}^{T}\left[|\dot{u}(t)|^{2}+\langle A(t) u(t), u(t)\rangle\right] d t & =\int_{0}^{T}\langle f(t), u(t)\rangle d t \\
& \leq\|f\|_{L^{r}[0, T]}\|u\|_{L^{r /(r-1)}[0, T]} \\
& \leq c\|f\|_{L^{r}[0, T]}\|u\|_{W^{1,2}[0, T]} \quad \text { (Sobolev inequality) }
\end{aligned}
$$


from which together with (3.1) we obtain $\|u\|_{W^{1,2}[0, T]} \leq C\|f\|_{L^{r}[0, T]}$ for some positive constant $C$ independent of $u$. This implies $A(t) u$ and $u \in L^{r}[0, T]$ and indeed

$$
\begin{aligned}
\|A(t) u\|_{L^{r}[0, T]}+\|u\|_{L^{r}[0, T]} & \leq\left(1+\alpha_{2}\right)\|u\|_{L^{r}[0, T]} \\
& \leq\left(1+\alpha_{2}\right) c_{1}\|u\|_{W^{1,2}[0, T]} \\
& \leq\left(1+\alpha_{2}\right) c_{1} C\|f\|_{L^{r}[0, T]}
\end{aligned}
$$

where $c_{1}$ comes from the embedding of $W^{1,2}[0, T]$ into $L^{r}[0, T]$. Let $C_{0}=\left(1+\alpha_{2}\right) c_{1} C$. It then follows that

$$
\begin{aligned}
\|u\|_{W_{\mathrm{per}}^{2, r}[0, T]} & =\|u\|_{L^{r}[0, T]}+\|\ddot{u}\|_{L^{r}[0, T]} \\
& \leq C_{0}\|f\|_{L^{r}[0, T]}+\|f-A(t) u\|_{L^{r}[0, T]} \\
& \leq\left(1+2 C_{0}\right)\|f\|_{L^{r}[0, T]}=\left(1+2 C_{0}\right)\|-\ddot{u}+A(t) u\|_{L^{r}[0, T]} \\
& =\left(1+2 C_{0}\right)\|u\|_{\mathrm{per}} .
\end{aligned}
$$

Therefore, by (3.2) and (3.3) we have

$$
\frac{1}{1+\alpha_{2}}\|u\|_{\mathrm{per}} \leq\|u\|_{W_{\mathrm{per}}^{2, r}[0, T]} \leq\left(1+2 C_{0}\right)\|u\|_{\mathrm{per}} .
$$

This proves part (2).

Proof of Theorem 1.1. First suppose that $u$ is a solution of (1.1), then $A(t) u(t)-$ $\ddot{u}(t)=\nabla \Phi(t, u(t))$. This implies that $u(t) \in \partial \Phi^{*}(t, A(t) u(t)-\ddot{u}(t))$, and therefore for $\eta \in W_{\text {per }}^{2, r}[0, T]$ we obtain

$$
\begin{aligned}
0 & =\int_{0}^{T}\langle A(t) u(t)-\ddot{u}(t)-\nabla \Phi(t, u(t)), \eta(t)\rangle d t \\
& =\int_{0}^{T}[\langle u(t), A(t) \eta(t)\rangle+\langle u(t), \ddot{\eta}(t)\rangle] d t-\int_{0}^{T}\langle\nabla \Phi(t, u(t)), \eta(t)\rangle d t
\end{aligned}
$$

thereby giving that $u$ is a critical point of $I$.

Now suppose that $u$ is a critical point of $I$, thus there exists $v \in \partial \Phi^{*}(t, A(t) u-\ddot{u})$ such that

$$
\int_{0}^{T}\langle v(t), A(t) \eta(t)-\ddot{\eta}(t)\rangle d t-\int_{0}^{T}\langle\nabla \Phi(t, u(t)), \eta(t)\rangle d t=0 \quad \text { for all } \eta \in W_{\mathrm{per}}^{2, r}[0, T] .
$$

Suppose that $w$ is the solution of the minimization problem $\inf _{w \in W^{1,2}[0, T], w(0)=w(T)} F(w)$ where

$$
F(w)=\frac{1}{2} \int_{0}^{T}\left[\langle w(t), A(t) w(t)\rangle+|\dot{w}(t)|^{2}\right] d t-\int_{0}^{T}\langle\nabla \Phi(t, u(t)), w(t)\rangle d t .
$$


It then follows that $A(t) w(t)-\ddot{w}(t)=\nabla \Phi(t, u(t))$ with $w(0)=w(T)$. It also follows that $\dot{w}(0)=\dot{w}(T)$. In fact, take an arbitrary $\xi \in \mathbb{R}^{N}$ and set $\eta_{0}(t)=\xi$. Note that $\eta_{0} \in W^{1,2}[0, T]$ and $\eta(0)=\eta(T)=\xi$. It follows that

$$
\begin{aligned}
0 & =\int_{0}^{T}\left\langle\nabla F(w), \eta_{0}\right\rangle d t \\
& =\int_{0}^{T}\left[\left\langle A(t) \eta_{0}(t), w(t)\right\rangle+\left\langle\dot{w}(t), \dot{\eta}_{0}(t)\right\rangle\right] d t-\int_{0}^{T}\left\langle\nabla \Phi(t, u(t)), \eta_{0}(t)\right\rangle d t \\
& =\int_{0}^{T}\left[\left\langle\eta_{0}(t), A(t) w(t)\right\rangle-\left\langle\ddot{w}(t), \eta_{0}(t)\right\rangle\right] d t+\left\langle\dot{w}(T), \eta_{0}(T)\right\rangle-\left\langle\dot{w}(0), \eta_{0}(0)\right\rangle \\
& -\int_{0}^{T}\left\langle\nabla \Phi(t, u(t)), \eta_{0}(t)\right\rangle d t \\
& =\int_{0}^{T}\left\langle\eta_{0}(t), A(t) w(t)-\ddot{w}(t)-\nabla \Phi(t, u(t))\right\rangle d t+\langle\dot{w}(T)-\dot{w}(0), \xi\rangle \\
& =\langle\dot{w}(T)-\dot{w}(0), \xi\rangle,
\end{aligned}
$$

from which and the fact that $\xi \in \mathbb{R}^{N}$ is arbitrary we indeed have $\dot{w}(0)=\dot{w}(T)$.

This and (3.4) together imply that

$$
\int_{0}^{T}\langle v(t), A(t) \eta(t)-\ddot{\eta}(t)\rangle d t=\int_{0}^{T}\langle A(t) w(t)-\ddot{w}(t), \eta(t)\rangle d t \quad \text { for all } \eta \in W_{\text {per }}^{2, r}[0, T] .
$$

It follows from $A(t) w(t)-\ddot{w}(t)=\nabla \Phi(t, u(t))$ and $v \in \partial \Phi^{*}(t, A(t) u(t)-\ddot{u}(t))$ that

$$
\begin{aligned}
\Phi^{*}(t, A(t) w(t)-\ddot{w}(t))+\Phi(t, u(t)) & =\langle A(t) w(t)-\ddot{w}(t), u(t)\rangle, \\
\Phi^{*}(t, A(t) u(t)-\ddot{u}(t))+\Phi(t, v(t)) & =\langle A(t) u(t)-\ddot{u}(t), v(t)\rangle .
\end{aligned}
$$

By adding (3.6) and (3.7) we obtain

$$
\begin{aligned}
\langle A(t) & w(t)-\ddot{w}(t), u(t)\rangle+\langle A(t) u(t)-\ddot{u}(t), v(t)\rangle \\
& =\Phi^{*}(t, A(t) w(t)-\ddot{w}(t))+\Phi(t, u(t))+\Phi^{*}(t, A(t) u(t)-\ddot{u}(t))+\Phi(t, v(t)) \\
& =\Phi^{*}(t, A(t) w(t)-\ddot{w}(t))+\Phi(t, v(t))+\Phi^{*}(t, A(t) u(t)-\ddot{u}(t))+\Phi(t, u(t)) \\
& \geq\langle A(t) w(t)-\ddot{w}(t), v(t)\rangle+\langle A(t) u(t)-\ddot{u}(t), u(t)\rangle,
\end{aligned}
$$

and consequently

$$
\begin{gathered}
\int_{0}^{T}\langle A(t) w(t)-\ddot{w}(t), u(t)\rangle d t+\int_{0}^{T}\langle A(t) u(t)-\ddot{u}(t), v(t)\rangle d t \\
\quad \geq \int_{0}^{T}\langle A(t) w(t)-\ddot{w}(t), v(t)\rangle d t+\int_{0}^{T}\langle A(t) u(t)-\ddot{u}(t), u(t)\rangle d t .
\end{gathered}
$$

It follows from (3.5) that

$$
\int_{0}^{T}\langle v(t), A(t) u(t)-\ddot{u}(t)\rangle d t=\int_{0}^{T}\langle A(t) w(t)-\ddot{w}(t), u(t)\rangle d t
$$


and

$$
\int_{0}^{T}\langle v(t), A(t) w(t)-\ddot{w}(t)\rangle d t=\int_{0}^{T}\langle A(t) w(t)-\ddot{w}(t), w(t)\rangle d t .
$$

Together with the previous inequality this yields that

$$
\begin{aligned}
\int_{0}^{T}\langle A(t) w(t)-\ddot{w}(t), u(t)\rangle d t+\int_{0}^{T}\langle A(t) w(t)-\ddot{w}(t), u(t)\rangle d t \\
\quad \geq \int_{0}^{T}\langle A(t) w(t)-\ddot{w}(t), w(t)\rangle d t+\int_{0}^{T}\langle A(t) u(t)-\ddot{u}(t), u(t)\rangle d t,
\end{aligned}
$$

and hence,

$$
\int_{0}^{T}\langle A(t) w(t)-\ddot{w}(t)-A(t) u(t)+\ddot{u}(t), w(t)-u(t)\rangle d t \leq 0 .
$$

On the other hand, since $\Lambda u=A(t) u-\ddot{u}(t)$ is a nonnegative operator we have that the latter is indeed zero,

$$
\int_{0}^{T}\langle A(t) w(t)-\ddot{w}(t)-A(t) u(t)+\ddot{u}(t), w(t)-u(t)\rangle d t=0 .
$$

This implies that the inequality in (3.9) is in fact an equality, which together with (3.8) implies that

$$
\begin{aligned}
0= & \int_{0}^{T}\left[\Phi^{*}(t, A(t) w(t)-\ddot{w}(t))+\Phi(t, v(t))-\langle A(t) w(t)-\ddot{w}(t), v(t)\rangle\right] d t \\
& +\int_{0}^{T}\left[\Phi^{*}(t, A(t) u(t)-\ddot{u}(t))+\Phi(t, u(t))-\langle A(t) u(t)-\ddot{u}(t), u(t)\rangle\right] d t .
\end{aligned}
$$

Together with the fact that

$$
\Phi^{*}(t, A(t) w(t)-\ddot{w}(t))+\Phi(t, v(t))-\langle A(t) w(t)-\ddot{w}(t), v(t)\rangle \geq 0
$$

and

$$
\Phi^{*}(t, A(t) u(t)-\ddot{u}(t))+\Phi(t, u(t))-\langle A(t) u(t)-\ddot{u}(t), u(t)\rangle \geq 0,
$$

this implies that $\Phi^{*}(t, A(t) u(t)-\ddot{u}(t))+\Phi(t, u(t))-\langle A(t) u(t)-\ddot{u}(t), u(t)\rangle=0$ and therefore $A(t) u(t)-\ddot{u}(t)=\nabla \Phi(t, u(t))$, and so $u$ is a solution of (1.1).

For simplicity of notation we will at times use the symbol $\Lambda$ to refer to the operator defined by $\Lambda u=A(t) u-\ddot{u}$.

Proof of Theorem 1.2. The existence of a critical point can be established by minimizing the functional $I$ on the Nehari manifold

$$
\mathcal{N}=\left\{u \in W_{\text {per }}^{2, p^{\prime}}[0, T] \mid \int_{0}^{T}\left\langle I^{\prime}(u(t)), u(t)\right\rangle d t=0, u \neq 0\right\} .
$$


We first prove that if $\|f\|_{L^{p^{\prime}}[0, T]}$ is small enough then there exists $r>0$ such that $\inf _{\|u\|_{\text {per }}=r} I(u)>I(0)$. We have

$$
\begin{gathered}
I(u)-I(0)=\frac{1}{p^{\prime}} \int_{0}^{T}|-\ddot{u}(t)+A(t) u(t)-f(t)|^{p^{\prime}} d t-\frac{1}{p} \int_{0}^{T}|u(t)|^{p} d t \\
-\int_{0}^{T}\langle f(t), u(t)\rangle d t-\frac{1}{p^{\prime}} \int_{0}^{T}|f(t)|^{p^{\prime}} d t .
\end{gathered}
$$

Let us first recall the elementary inequality

$$
|a|^{p^{\prime}} \leq 2^{p^{\prime}-1}\left(|a-b|^{p^{\prime}}+|b|^{p^{\prime}}\right) \quad \text { for all } a, b \in \mathbb{R}^{N},
$$

from which we obtain

$$
|a-b|^{p^{\prime}} \geq 2^{1-p^{\prime}}|a|^{p^{\prime}}-|b|^{p^{\prime}} \quad \text { for all } a, b \in \mathbb{R}^{N} .
$$

It follows from this inequality that

$$
\begin{aligned}
I(u)-I(0) \geq & \frac{2^{1-p^{\prime}}}{p^{\prime}} \int_{0}^{T}|\Lambda u(t)|^{p^{\prime}} d t-\frac{1}{p^{\prime}} \int_{0}^{T}|f(t)|^{p^{\prime}} d t-\frac{1}{p} \int_{0}^{T}|u(t)|^{p} d t \\
& \quad-\int_{0}^{T} f(t) u(t) d t-\frac{1}{p^{\prime}} \int_{0}^{T}|f(t)|^{p^{\prime}} d t \\
& \geq \frac{2^{1-p^{\prime}}}{p^{\prime}} \int_{0}^{T}|\Lambda u(t)|^{p^{\prime}} d t-\frac{3}{p^{\prime}} \int_{0}^{T}|f(t)|^{p^{\prime}} d t-\frac{2}{p} \int_{0}^{T}|u(t)|^{p} d t \\
& =\frac{2^{1-p^{\prime}}}{p^{\prime}}\|u\|_{\mathrm{per}}^{p^{\prime}}-\frac{2}{p}\|u\|_{L^{p}[0, T]}^{p}-\frac{3}{p^{\prime}} \int_{0}^{T}|f(t)|^{p^{\prime}} d t \\
& \geq \frac{2^{1-p^{\prime}}}{p^{\prime}}\|u\|_{\text {per }}^{p^{\prime}}-C_{0}\|u\|_{\text {per }}^{p}-\frac{3}{p^{\prime}} \int_{0}^{T}|f(t)|^{p^{\prime}} d t \quad \text { (Sobolev inequality) }
\end{aligned}
$$

where $C_{0}>$ comes from the embedding of $W_{\text {per }}^{2, p^{\prime}}[0, T]$ into $L^{p}[0, T]$. Note that since $p>p^{\prime}$, if $\|u\|_{\text {per }}=r$ for some $r>0$ small enough, then there exists $\rho>0$ depending only on $p$ and $T$ such that

$$
\frac{2^{1-p^{\prime}}}{p^{\prime}}\|u\|_{\mathrm{per}}^{p^{\prime}}-C_{0}\|u\|_{\mathrm{per}}^{p}>\rho .
$$

Thus, if $\left(3 / p^{\prime}\right) \int_{0}^{T}|f(t)|^{p^{\prime}} d t<\rho / 2$ then for $\|u\|_{\text {per }}=r$ we have $I(u)-I(0)>\rho / 2$.

Let $\left\{u_{n}\right\}_{n \in \mathbb{N}}$ be a minimizing sequence for $I$ over the Nehari manifold $\mathcal{N}$. Then

$$
I\left(u_{n}\right)=I\left(u_{n}\right)-\frac{1}{p} \int_{0}^{T}\left\langle I^{\prime}\left(u_{n}(t)\right), u_{n}(t)\right\rangle d t .
$$


Expanding the right-hand side,

$$
\begin{aligned}
I\left(u_{n}\right)=\frac{1}{p^{\prime}} & \int_{0}^{T}\left|\Lambda u_{n}(t)-f(t)\right|^{p^{\prime}} d t-\frac{1}{p} \int_{0}^{T}\left|u_{n}(t)\right|^{p} d t-\int_{0}^{T}\left\langle f(t), u_{n}(t)\right\rangle d t \\
& -\frac{1}{p} \int_{0}^{T}\left|\Lambda u_{n}(t)-f(t)\right|^{p^{\prime}-2}\left\langle\Lambda u_{n}(t)-f(t), \Lambda u_{n}(t)\right\rangle d t \\
& +\frac{1}{p} \int_{0}^{T}\left|u_{n}(t)\right|^{p} d t+\frac{1}{p} \int_{0}^{T}\left\langle f(t), u_{n}(t)\right\rangle d t,
\end{aligned}
$$

thereby giving that

$$
\begin{gathered}
I\left(u_{n}\right)=\left(\frac{1}{p^{\prime}}-\frac{1}{p}\right) \int_{0}^{T}\left|\Lambda u_{n}(t)-f(t)\right|^{p^{\prime}} d t-\frac{1}{p^{\prime}} \int_{0}^{T}\left\langle f(t), u_{n}(t)\right\rangle d t \\
-\frac{1}{p} \int_{0}^{T}\left|\Lambda u_{n}(t)-f(t)\right|^{p^{\prime}-2}\left\langle\Lambda u_{n}(t)-f(t), f(t)\right\rangle d t .
\end{gathered}
$$

It follows from Hölder's inequality that

$$
\begin{aligned}
I\left(u_{n}\right) \geq & \left(\frac{1}{p^{\prime}}-\frac{1}{p}\right) \int_{0}^{T}\left|\Lambda u_{n}(t)-f(t)\right|^{p^{\prime}} d t-\frac{1}{p^{\prime}}\|f\|_{L^{p^{\prime}}[0, T]}\left\|u_{n}\right\|_{L^{p}[0, T]} \\
& \quad-\frac{1}{p}\left(\int_{0}^{T}\left(\left|\Lambda u_{n}(t)-f(t)\right|^{p^{\prime}-1}\right)^{p^{\prime} /\left(p^{\prime}-1\right)} d t\right)^{\left(p^{\prime}-1\right) / p^{\prime}}\left(\int_{0}^{T}|f(t)|^{p^{\prime}} d t\right)^{1 / p^{\prime}} \\
= & \left(\frac{1}{p^{\prime}}-\frac{1}{p}\right)\left\|\Lambda u_{n}-f\right\|_{L^{p^{\prime}[0, T]}}^{p^{\prime}}-\frac{1}{p^{\prime}}\|f\|_{L^{p^{\prime}}[0, T]}\left\|u_{n}\right\|_{L^{p}[0, T]} \\
& \quad-\frac{1}{p}\left\|\Lambda u_{n}-f\right\|_{L^{p^{\prime}[0, T]}}^{p^{\prime}-1}\|f\|_{L^{p^{\prime}}[0, T]} \\
\geq & \left(\frac{1}{p^{\prime}}-\frac{1}{p}\right)\left\|u_{n}\right\|_{\text {per }}-\left.\|f\|_{L^{p^{\prime}[0, T]}}\right|^{p^{\prime}}-\frac{C}{p^{\prime}}\|f\|_{\left.L^{p^{\prime}[0, T]}\right]}\left\|u_{n}\right\|_{\text {per }} \\
& \quad \frac{1}{p}\left(\left\|u_{n}\right\|_{\text {per }}+\|f\|_{\left.L^{p^{\prime}[0, T]}\right)^{p^{\prime}-1}\|f\|_{L^{p^{\prime}[0, T]},},}\right.
\end{aligned}
$$

where the last inequality is a consequence of Hölder's inequality and the continuous embedding $W_{\text {per }}^{2, p^{\prime}}[0, T] \hookrightarrow L^{p}[0, T]$. The right-hand side can now be seen to be coercive since $p^{\prime}>1$ and $\left(1 / p^{\prime}\right)-(1 / p)>0$. I $\left(u_{n}\right)$ is bounded from above, hence it follows that the sequence $\left\{u_{n}\right\}_{n \in \mathbb{N}}$ is bounded in $W_{\text {per }}^{2, p^{\prime}}[0, T]$. By the Banach-Alaoglu theorem, $\left\{u_{n}\right\}_{n \in \mathbb{N}}$ is contained in a weakly compact subset of $W_{\text {per }}^{2, p^{\prime}}[0, T]$, and hence there is a function $u \in W_{\text {per }}^{2, p^{\prime}}[0, T]$ such that, up to a subsequence, $u_{n}$ converges weakly to $u$. Additionally, since $W_{\text {per }}^{2, p^{\prime}}[0, T]$ can be compactly embedded into $L^{p}[0 T]$, it follows that $u_{n}$ converges strongly to $u$ in $L^{p}[0, T]$. Defining the functional $G$ by the constraint

$$
G(u)=\int_{0}^{T}\left\langle I^{\prime}(u(t)), u(t)\right\rangle d t=0
$$


and using the method of Lagrange multipliers,

$$
I^{\prime}\left(u_{n}\right)=\lambda_{n} G^{\prime}\left(u_{n}\right)+o(n)
$$

where $\lambda_{n} \rightarrow 0$, and therefore $I^{\prime}\left(u_{n}\right) \rightarrow 0$ taking into account the boundedness of $\left\{u_{n}\right\}_{n \in \mathbb{N}}$. Now it follows that

$$
\begin{aligned}
\int_{0}^{T}\left\langle I^{\prime}\left(u_{n}(t)\right), u(t)\right\rangle d t= & \int_{0}^{T}\left|\Lambda u_{n}(t)-f(t)\right|^{p^{\prime}-2}\left\langle\Lambda u_{n}(t)-f(t), \Lambda u\right\rangle d t \\
& -\int_{0}^{T}\left|u_{n}(t)\right|^{p-2}\left\langle u_{n}(t), u(t)\right\rangle d t-\int_{0}^{T} f(t) u(t) d t \rightarrow 0
\end{aligned}
$$

which implies that

$$
\int_{0}^{T}\left|\Lambda u_{n}(t)-f(t)\right|^{p^{\prime}-2}\left\langle\Lambda u_{n}(t)-f(t), \Lambda u(t)\right\rangle d t \rightarrow \int_{0}^{T}|u(t)|^{p} d t+\int_{0}^{T} f(t) u(t) d t .
$$

Similarly,

$$
\begin{gathered}
\int_{0}^{T}\left\langle I^{\prime}\left(u_{n}(t)\right), u_{n}(t)\right\rangle d t=\int_{0}^{T}\left|\Lambda u_{n}(t)-f(t)\right|^{p^{\prime}-2}\left\langle\Lambda u_{n}(t)-f(t), \Lambda u_{n}(t)\right\rangle d t \\
-\int_{0}^{T}\left|u_{n}(t)\right|^{p} d t-\int_{0}^{T} f(t) u_{n}(t) d t \rightarrow 0
\end{gathered}
$$

and hence,

$$
\int_{0}^{T}\left|\Lambda u_{n}(t)-f(t)\right|^{p^{\prime}-2}\left\langle\Lambda u_{n}(t)-f(t), \Lambda u_{n}(t)\right\rangle d t \rightarrow \int_{0}^{T}|u(t)|^{p} d t+\int_{0}^{T} f(t) u(t) d t .
$$

The functional $\int_{0}^{T}|\Lambda u(t)-f(t)|^{p^{\prime}} d t$ is lower semi-continuous, that is,

$$
\int_{0}^{T}|\Lambda u(t)-f(t)|^{p^{\prime}} d t \leq \liminf _{n \rightarrow \infty} \int_{0}^{T}\left|\Lambda u_{n}(t)-f(t)\right|^{p^{\prime}} d t .
$$

In addition, the functional is convex, therefore,

$$
\begin{aligned}
\int_{0}^{T}\left|\Lambda u_{n}(t)-f(t)\right|^{p^{\prime}} d t \leq & \int_{0}^{T}|\Lambda u(t)-f(t)|^{p^{\prime}} d t \\
& +\int_{0}^{T}\left|\Lambda u_{n}(t)-f(t)\right|^{p^{\prime}-2}\left\langle\Lambda u_{n}(t)-f(t), \Lambda u_{n}(t)\right\rangle d t \\
& -\int_{0}^{T}\left|\Lambda u_{n}(t)-f(t)\right|^{p^{\prime}-2}\left\langle\Lambda u_{n}(t)-f(t), \Lambda u(t)\right\rangle d t
\end{aligned}
$$

where the latter terms cancel in the limit from (3.10) and (3.11). Therefore,

$$
\int_{0}^{T}|\Lambda u(t)-f(t)|^{p^{\prime}} d t \geq \limsup _{n \rightarrow \infty} \int_{0}^{T}\left|\Lambda u_{n}(t)-f(t)\right|^{p^{\prime}} d t
$$


Inequalities (3.12) and (3.13) together then imply that

$$
\lim _{n \rightarrow \infty} \int_{0}^{T}\left|\Lambda u_{n}(t)-f(t)\right|^{p^{\prime}} d t=\int_{0}^{T}|\Lambda u(t)-f(t)|^{p^{\prime}} d t,
$$

that is to say, $\left\|\Lambda u_{n}-f\right\|_{L^{p^{\prime}}} \rightarrow\|\Lambda u-f\|_{L^{p^{\prime}}}$. Therefore $\Lambda u_{n} \rightarrow \Lambda u$ strongly in $L^{p^{\prime}}[0, T]$ which then implies $u_{n} \rightarrow u$ strongly in $W_{\text {per }}^{2, p^{\prime}}[0, T]$. Since $I$ and $I^{\prime}$ are continuous, $u$ is then a nontrivial critical point of $I$. By Theorem $1.1 u$ is then a solution of Equation (1.4).

Proof of Theorem 1.3. First suppose that $u$ is a solution of (1.2), that is,

$$
\left\{\begin{array}{l}
A(t) u(t)-\ddot{u}(t)=\nabla \Phi(t, u(t)), \\
\dot{u}(0)=-\nabla \Psi_{1}(u(0)), \\
\dot{u}(T)=\nabla \Psi_{2}(u(T)) .
\end{array}\right.
$$

This implies that $u(t) \in \partial \Phi^{*}(A(t) u(t)-\ddot{u}(t)), u(0) \in \partial \Psi_{1}^{*}(-\dot{u}(0))$, and $u(T) \in \partial \Psi_{2}^{*}(\dot{u}(T))$. For $\eta \in W^{2, r}[0, T]$, the 'Green formula' yields

$$
\begin{aligned}
\int_{0}^{T} & {[\langle u(t), A(t) \eta(t)\rangle-\langle u(t), \ddot{\eta}(t)\rangle] d t-\int_{0}^{T}\langle\nabla \Phi(t, u(t)), \eta(t)\rangle d t } \\
+ & \langle u(0),-\dot{\eta}(0)\rangle+\langle u(T), \dot{\eta}(T)\rangle-\left\langle\nabla \Psi_{1}(u(0)), \eta(0)\right\rangle-\left\langle\nabla \Psi_{2}(u(T)), \eta(T)\right\rangle \\
= & \int_{0}^{T}[\langle A(t) u(t)-\ddot{u}(t)-\nabla \Phi(t, u(t)), \eta(t)\rangle] d t-\left\langle\dot{u}(0)+\nabla \Psi_{1}(u(0)), \eta(0)\right\rangle \\
\quad & \quad\left\langle\dot{u}(T)-\nabla \Psi_{2}(u(T)), \eta(T)\right\rangle=0 .
\end{aligned}
$$

Therefore, $u$ is a critical point of $I$.

Suppose that $u$ is a critical point of $I$, and thus there exist $v(t) \in \partial \Phi^{*}(A(t) u(t)-\ddot{u}(t))$, $w_{1} \in \partial \Psi_{1}(-\dot{u}(0))$ and $w_{2} \in \partial \Psi_{2}(\dot{u}(T))$ such that

$$
\begin{aligned}
& \int_{0}^{T}[\langle v(t), A(t) \eta(t)-\ddot{\eta}(t)\rangle] d t-\int_{0}^{T}\langle\nabla \Phi(t, u(t)), \eta(t)\rangle d t \\
& \quad+\left\langle w_{1},-\dot{\eta}(0)\right\rangle+\left\langle w_{2}, \dot{\eta}(T)\right\rangle-\left\langle\nabla \Psi_{1}(u(0)), \eta(0)\right\rangle-\left\langle\nabla \Psi_{2}(u(T)), \eta(T)\right\rangle=0
\end{aligned}
$$

for all $\eta \in W^{2, r}[0, T]$. Let $x \in W^{1,2}[0, T]$ be a solution of the minimizing problem

$$
\begin{aligned}
\inf _{x \in W^{1,2}[0, T]}\left\{\frac{1}{2} \int_{0}^{T}\left[\langle x(t), A(t) x(t)\rangle+|\dot{x}(t)|^{2}-2\langle x(t), \nabla \Phi(t, u(t))\rangle\right] d t\right. \\
\left.+\left\langle x(T), \nabla \Psi_{2}(u(T))\right\rangle+\left\langle x(0), \nabla \Psi_{1}(u(0))\right\rangle\right\} .
\end{aligned}
$$

It follows that $x$ is a solution of the system

$$
\left\{\begin{array}{l}
A(t) x(t)-\ddot{x}(t)=\nabla \Phi(t, u(t)), \\
\dot{x}(0)=-\nabla \Psi_{1}(u(0)), \\
\dot{x}(T)=\nabla \Psi_{2}(u(T)) .
\end{array}\right.
$$


This together with (3.14) implies that

$$
\begin{aligned}
& \int_{0}^{T}\langle v(t), A(t) \eta(t)-\ddot{\eta}(t)\rangle d t-\int_{0}^{T}\langle A(t) x(t)-\ddot{x}(t), \eta(t)\rangle d t \\
& \quad+\left\langle w_{1},-\dot{\eta}(0)\right\rangle+\left\langle w_{2}, \dot{\eta}(T)\right\rangle+\langle\dot{x}(0), \eta(0)\rangle-\langle\dot{x}(T), \eta(T)\rangle=0,
\end{aligned}
$$

for all $\eta \in W^{2, r}[0, T]$. It follows from the above and integration by parts that

$$
\int_{0}^{T}\langle v(t)-x(t), A(t) \eta(t)-\ddot{\eta}(t)\rangle d t+\left\langle w_{1}-x(0), \dot{\eta}(0)\right\rangle+\left\langle w_{2}-x(T), \dot{\eta}(T)\right\rangle=0,
$$

for all $\eta \in W^{2, r}[0, T]$. As seen in (3.15), the operator $\mathcal{A}: W^{2, r^{\prime}}[0, T] \rightarrow L^{r^{\prime}}[0, T] \times$ $\mathbb{R}^{N} \times \mathbb{R}^{N}$ defined by $\mathcal{A}(x)=(A(t) x(t)-\ddot{x}(t), \dot{x}(0), \dot{x}(T))$ is onto. This together with the above equation implies that $x(t)=v(t)$. Therefore,

$$
\left\{\begin{array}{l}
x(0) \in \partial \Psi_{1}(-\dot{u}(0)) \\
x(T) \in \partial \Psi_{2}(\dot{u}(T)) \\
\dot{x}(0)=-\nabla \Psi_{1}(u(0)) \\
\dot{x}(T)=\nabla \Psi_{2}(u(T))
\end{array}\right.
$$

from which we obtain

$$
\begin{aligned}
\langle u(0),- & \dot{x}(0)\rangle+\langle u(T), \dot{x}(T)\rangle+\langle x(0),-\dot{u}(0)\rangle+\langle x(T), \dot{u}(T)\rangle \\
= & \Psi_{1}^{*}(-\dot{x}(0))+\Psi_{2}^{*}(\dot{x}(T))+\Psi_{1}(u(0))+\Psi_{2}(u(T))+\Psi_{1}^{*}(-\dot{u}(0))+\Psi_{2}^{*}(\dot{u}(T)) \\
& +\Psi_{1}(x(0))+\Psi_{2}(x(T)) \\
= & \Psi_{1}^{*}(-\dot{x}(0))+\Psi_{2}^{*}(\dot{x}(T))+\Psi_{1}(x(0))+\Psi_{2}(x(T))+\Psi_{1}^{*}(-\dot{u}(0))+\Psi_{2}^{*}(\dot{u}(T)) \\
& +\Psi_{1}(u(0))+\Psi_{2}(u(T)) \\
\geq & \langle x(0),-\dot{x}(0)\rangle+\langle x(T), \dot{x}(T)\rangle+\langle u(0),-\dot{u}(0)\rangle+\langle u(T), \dot{u}(T)\rangle .
\end{aligned}
$$

From this inequality we deduce that

$$
\langle u(0)-x(0), \dot{x}(0)-\dot{u}(0)\rangle-\langle u(T)-x(T), \dot{x}(T)-\dot{u}(T)\rangle \leq 0 .
$$

By the same argument as (3.8) in the proof of Theorem 1.1, it follows from $x(t)=$ $v(t) \in \partial \Phi^{*}(t, A(t) u(t)-\ddot{u}(t))$ and $A(t) x(t)-\ddot{x}(t)=\nabla \Phi(t, u(t))$ that

$$
\int_{0}^{T}\langle A(t) x(t)-\ddot{x}(t)-A(t) u(t)+\ddot{u}(t), x(t)-u(t)\rangle d t \leq 0 .
$$

Taking the sum of inequalities (3.17) and (3.18) and using integration by parts, we have

$$
\int_{0}^{T}\langle A(t) x(t)-A(t) u(t), x(t)-u(t)\rangle d t+\int_{0}^{T}|\dot{x}(t)-\dot{u}(t)|^{2} d t \leq 0
$$


On the other hand, the operator $A(t)$ is strictly positive definite, which implies that the latter holds with equality. This implies that $x=u$ and therefore $u$ is a solution of

$$
\left\{\begin{array}{l}
A(t) u(t)-\ddot{u}(t)=\nabla \Phi(t, u(t)), \\
\dot{u}(0)=-\nabla \Psi_{1}(u(0)), \\
\dot{u}(T)=\nabla \Psi_{2}(u(T)) .
\end{array}\right.
$$

This concludes the proof.

REMARK 3.2. The preceding proof can be easily modified to deal with other types of boundary conditions, including the case of

$$
\left\{\begin{array}{l}
u(0)=\nabla \Psi_{1}(\dot{u}(0)), \\
u(T)=-\nabla \Psi_{2}(\dot{u}(T)) .
\end{array}\right.
$$

Proof of Theorem 1.4. We first consider the case of $p=q$. We note that $I(0)=0$. Since $\Phi$ is lower semi-continuous, strictly convex, and coercive it follows that the mapping $u \mapsto \Phi^{*}(\cdot, u)$ is continuously differentiable. Similarly $\Psi_{1}^{*}, \Psi_{2}^{*} \in C^{1}\left(\mathbb{R}^{N}\right)$, which implies that $I: W^{2, p^{\prime}}[0, T] \rightarrow \mathbb{R}$ is continuously differentiable. Condition (i) of the theorem implies the existence of constants $c, c_{1}, c_{2}>0$ such that for all $t \in[0, T]$ and $\xi \in \mathbb{R}^{N}$,

$$
\begin{aligned}
c\left(|\xi|^{p}-1\right) & \leq \Phi(t, \xi), \\
c_{i}\left(|\xi|^{p_{i}}-1\right) & \leq \Psi_{i}(\xi) .
\end{aligned}
$$

Combined with condition (ii), this implies the existence of constants $\alpha^{*}, c^{*}, \alpha_{i}^{*}, c_{i}^{*}>0$, such that for all $t \in[0, T], \xi \in \mathbb{R}^{N}$ and $i=1,2$,

$$
\begin{aligned}
& \alpha^{*}|\xi|^{p^{\prime}} \leq \Phi^{*}(t, \xi) \leq c^{*}\left(|\xi|^{p^{\prime}}+1\right), \\
& \alpha_{i}^{*}|\xi|^{q_{i}^{\prime}} \leq \Psi_{i}^{*}(\xi) \leq c_{i}^{*}\left(|\xi|^{p_{i}^{\prime}}+1\right),
\end{aligned}
$$

where $p_{i}, p_{i}^{\prime}$ and $q_{i}, q_{i}^{\prime}$ are conjugate pairs. For $\lambda>0$ we have

$$
\begin{aligned}
& I(\lambda u) \leq c^{*} \lambda^{p^{\prime}}\|\Lambda u\|_{L^{p^{\prime}}[0, T]}^{p^{\prime}}-c \lambda^{p}\|u\|_{L^{p}[0, T]}^{p} \\
& \quad+c_{1}^{*} \lambda^{p_{1}^{\prime}}|\dot{u}(0)|^{p_{1}^{\prime}}+c_{2}^{*} \lambda^{p_{2}^{\prime}}|\dot{u}(T)|^{p_{2}^{\prime}}-c_{1} \lambda^{p_{1}}|u(0)|^{p_{1}}-c_{2} \lambda^{p_{2}}|u(T)|^{p_{2}}+C,
\end{aligned}
$$

for some constant $C \in \mathbb{R}$. Since $p>2>p^{\prime}, p_{1}^{\prime}, p_{2}^{\prime}$ we note that $I(\lambda u) \rightarrow-\infty$ as $\lambda \rightarrow \infty$, which implies the existence of a function $e \in W^{2, p^{\prime}}[0, T]$ such that $I(e)<0$, as we can simply take any function with a sufficiently large norm in $L^{p}[0, T]$. Similarly, we have the following inequality in the opposite direction,

$$
\begin{gathered}
I(u) \geq C_{0}\|u\|_{W^{2, p^{\prime}}[0, T]}^{p^{\prime}}-\alpha\|u\|_{L^{p}[0, T]}^{p}+\alpha_{1}^{*}|\dot{u}(0)|^{q_{1}^{\prime}}+\alpha_{2}^{*}|\dot{u}(T)|^{q_{2}^{\prime}} \\
\quad-\alpha_{1}|u(0)|^{q_{1}}-\alpha_{2}|u(T)|^{q_{2}} \\
\geq C_{0}\|u\|_{W^{2, p^{\prime}[0, T]}}^{p^{\prime}}-C\|u\|_{W^{2, p^{\prime}}[0, T]}^{p}+\alpha_{1}^{*}|\dot{u}(0)|^{q_{1}^{\prime}}+\alpha_{2}^{*}|\dot{u}(T)|^{q_{2}^{\prime}} \\
\quad-C_{1}\|u\|_{W^{2, p^{\prime}}[0, T]}^{q_{1}}-C_{2}\|u\|_{W^{2, p^{\prime}}[0, T]}^{q_{2}},
\end{gathered}
$$


for some $C, C_{0}, C_{1}, C_{2}>0$, making use of the fact that $W^{2, p^{\prime}}[0, T]$ can be continuously (indeed compactly) embedded in both $C[0, T]$ and $L^{p}[0, T]$. Noting that $q, q_{1}$, $q_{2}>2>p^{\prime}$, it then follows that

$$
\inf _{\|u\|=r} I(u)>0
$$

for $r$ sufficiently close to the origin. Hence by the mountain pass theorem, there exists a sequence $\left\{u_{n}\right\}_{n \in \mathbb{N}}$ with $u_{n} \in W^{2, p^{\prime}}[0, T]$ for all $n$, satisfying

$$
\lim _{n \rightarrow \infty} I\left(u_{n}\right)=c=\inf _{\gamma \in \Gamma} \max _{t \in[0,1]} I(\gamma(t))
$$

and

$$
\lim _{n \rightarrow \infty} I^{\prime}\left(u_{n}\right)=0
$$

where

$$
\Gamma=\left\{\gamma \in C\left([0,1], W^{2, p^{\prime}}[0, T]\right): \gamma(0)=0, \gamma(1)=e\right\} .
$$

It is easily seen that $c>0$. It follows immediately that

$$
I\left(u_{n}\right)-\frac{1}{\rho} \int_{0}^{T}\left\langle I^{\prime}\left(u_{n}(t)\right), u_{n}(t)\right\rangle d t \rightarrow c,
$$

where $\rho$ is chosen such that $p^{\prime}, p_{i}^{\prime}<\rho<2$. Since $\Phi$ is convex and differentiable, it follows that

$$
v=\nabla \Phi(t, u) \Leftrightarrow u=\nabla \Phi^{*}(t, v) \Leftrightarrow \Phi^{*}(t, v)=\langle u, v\rangle-\Phi(t, u) .
$$

Hence condition (2) implies that

$$
\Phi^{*}(t, v)=\langle u, v\rangle-\Phi(t, u) \geq\left(1-\frac{1}{p}\right)\langle u, v\rangle=\frac{1}{p^{\prime}}\left\langle\nabla \Phi^{*}(t, v), v\right\rangle .
$$

Similarly, it follows that

$$
\Psi_{i}^{*}(u) \geq \frac{1}{p_{i}^{\prime}}\left\langle\nabla \Psi_{i}^{*}(u), u\right\rangle \quad \text { for } i=1,2 .
$$

Now expanding the left-hand side of (3.23),

$$
\begin{aligned}
I\left(u_{n}\right)- & \frac{1}{\rho} \int_{0}^{T}\left\langle I^{\prime}\left(u_{n}(t)\right), u_{n}(t)\right\rangle d t \\
= & \int_{0}^{T}\left[\Phi^{*}\left(t, \Lambda u_{n}(t)\right)-\Phi\left(t, u_{n}(t)\right)\right] d t+\Psi_{1}^{*}\left(-\dot{u}_{n}(0)\right)+\Psi_{2}^{*}\left(\dot{u}_{n}(T)\right)-\Psi_{1}\left(u_{n}(0)\right) \\
& -\Psi_{2}\left(u_{n}(T)\right)-\frac{1}{\rho} \int_{0}^{T}\left[\left\langle\nabla \Phi^{*}\left(t, \Lambda u_{n}(t)\right), \Lambda u_{n}(t)\right\rangle-\left\langle\nabla \Phi\left(t, u_{n}(t)\right), u_{n}(t)\right\rangle\right] d t \\
& +\frac{1}{\rho}\left\langle\nabla \Psi_{1}^{*}\left(-\dot{u}_{n}(0)\right), \dot{u}_{n}(0)\right\rangle-\frac{1}{\rho}\left\langle\nabla \Psi_{2}^{*}\left(\dot{u}_{n}(T)\right), \dot{u}_{n}(T)\right\rangle+\frac{1}{\rho}\left\langle\nabla \Psi_{1}\left(u_{n}(0)\right), u_{n}(0)\right\rangle \\
& +\frac{1}{\rho}\left\langle\nabla \Psi_{2}\left(u_{n}(T)\right), u_{n}(T)\right\rangle .
\end{aligned}
$$


From inequalities (3.20), (3.21), (3.24) and (3.25) and condition (ii) of the theorem we then have

$$
\begin{aligned}
I\left(u_{n}\right)- & \frac{1}{\rho} \int_{0}^{T}\left\langle I^{\prime}\left(u_{n}(t)\right), u_{n}(t)\right\rangle d t \\
\geq & \left(1-\frac{p^{\prime}}{\rho}\right) \alpha^{*}\left\|\Lambda u_{n}\right\|_{L^{p^{\prime}[0, T]}}^{q^{\prime}}+\left(\frac{p}{\rho}-1\right) c\left\|u_{n}\right\|_{L^{p}[0, T]}^{p}+\left(1-\frac{p_{1}^{\prime}}{\rho}\right) \alpha_{1}^{*}|\dot{u}(0)|^{q_{1}^{\prime}} \\
& +\left(1-\frac{p_{2}^{\prime}}{\rho}\right) \alpha_{2}^{*}|\dot{u}(T)|^{q_{2}^{\prime}}+\left(\frac{p_{1}}{\rho}-1\right) c_{1}\left(u_{n}(0)\right)^{p_{1}}+\left(\frac{p_{2}}{\rho}-1\right) c_{2}\left(u_{n}(T)\right)^{p_{2}}+C,
\end{aligned}
$$

for some constant $C \in \mathbb{R}$. Since $p^{\prime}, p_{i}^{\prime}<\rho<2<p, p_{i}$, all coefficients on the right-hand side are positive. The right side is hence coercive and (3.23) then implies that each term is bounded. In particular, $\left\{\Lambda u_{n}\right\}_{n \in \mathbb{N}}$ is bounded in $L^{p^{\prime}}[0, T]$ hence $u_{n}$ is bounded in $W^{2, p^{\prime}}[0, T]$. Thus, by the Banach-Alaoglu theorem, the sequence $\left\{u_{n}\right\}_{n \in \mathbb{N}}$ is contained in a weakly compact subset of $W^{2, p^{\prime}}[0, T]$, and hence there is a function $u \in W^{2, p^{\prime}}[0, T]$ such that, up to a subsequence, $u_{n}$ converges weakly to $u$. Now since $I^{\prime}\left(u_{n}\right) \rightarrow 0$,

$$
\begin{aligned}
\int_{0}^{T}\left\langle I^{\prime}\left(u_{n}(t)\right), u_{n}(t)\right\rangle d t=\int_{0}^{T} & {\left[\left\langle\nabla \Phi^{*}\left(t, \Lambda u_{n}(t)\right), \Lambda u_{n}(t)\right\rangle-\left\langle\nabla \Phi\left(t, u_{n}(t)\right), u_{n}(t)\right\rangle\right] d t } \\
& -\left\langle\nabla \Psi_{1}^{*}\left(-\dot{u}_{n}(0)\right), \dot{u}_{n}(0)\right\rangle+\left\langle\nabla \Psi_{2}^{*}\left(\dot{u}_{n}(T)\right), \dot{u}_{n}(T)\right\rangle \\
& -\left\langle\nabla \Psi_{1}\left(u_{n}(0)\right), u_{n}(0)\right\rangle-\left\langle\nabla \Psi_{2}\left(u_{n}(T)\right), u_{n}(T)\right\rangle \rightarrow 0 .
\end{aligned}
$$

Since $W^{2, p^{\prime}}[0, T]$ can be compactly embedded in $L^{p}[0, T]$ and $C^{1}[0, T]$, it follows that $u_{n}$ converges strongly to $u$ in $L^{p}[0, T]$, and $u_{n}$ and its derivative converge pointwise (indeed, uniformly). This implies that

$$
\begin{aligned}
\int_{0}^{T}\left\langle\nabla \Phi^{*}\left(t, \Lambda u_{n}(t)\right), \Lambda u_{n}(t)\right\rangle d t & \\
\rightarrow & \int_{0}^{T}\langle\nabla \Phi(t, u(t)), u(t)\rangle d t+\left\langle\nabla \Psi_{1}^{*}(-\dot{u}(0)), \dot{u}(0)\right\rangle-\left\langle\nabla \Psi_{2}^{*}(\dot{u}(T)), \dot{u}(T)\right\rangle \\
\quad & +\left\langle\nabla \Psi_{1}(u(0)), u(0)\right\rangle+\left\langle\nabla \Psi_{2}(u(T)), u(T)\right\rangle .
\end{aligned}
$$

Similarly, we have

$$
\begin{aligned}
\int_{0}^{T}\left\langle I^{\prime}\left(u_{n}(t)\right), u(t)\right\rangle d t=\int_{0}^{T} & {\left[\left\langle\nabla \Phi^{*}\left(t, \Lambda u_{n}(t)\right), \Lambda u(t)\right\rangle-\left\langle\nabla \Phi\left(t, u_{n}(t)\right), u(t)\right\rangle\right] d t } \\
& -\left\langle\nabla \Psi_{1}^{*}\left(-\dot{u}_{n}(0)\right), \dot{u}(0)\right\rangle+\left\langle\nabla \Psi_{2}^{*}\left(\dot{u}_{n}(T)\right), \dot{u}(T)\right\rangle \\
& -\left\langle\nabla \Psi_{1}\left(u_{n}(0)\right), u(0)\right\rangle-\left\langle\nabla \Psi_{2}\left(u_{n}(T)\right), u(T)\right\rangle \rightarrow 0,
\end{aligned}
$$

from which we conclude that

$$
\begin{aligned}
\int_{0}^{T}\left\langle\nabla \Phi^{*}\left(t, \Lambda u_{n}(t)\right), \Lambda u(t)\right\rangle d t \\
\quad \rightarrow \int_{0}^{T}\langle\nabla \Phi(t, u(t)), u(t)\rangle d t+\left\langle\nabla \Psi_{1}^{*}(-\dot{u}(0)), \dot{u}(0)\right\rangle-\left\langle\nabla \Psi_{2}^{*}(\dot{u}(T)), \dot{u}(T)\right\rangle \\
\quad+\left\langle\nabla \Psi_{1}(u(0)), u(0)\right\rangle+\left\langle\nabla \Psi_{2}(u(T)), u(T)\right\rangle
\end{aligned}
$$


The functional $\int_{0}^{T} \Phi^{*}(t, \Lambda u(t)) d t$ is lower semi-continuous, hence

$$
\int_{0}^{T} \Phi^{*}(t, \Lambda u(t)) d t \leq \liminf _{n \rightarrow \infty} \int_{0}^{T} \Phi^{*}\left(t, \Lambda u_{n}(t)\right) d t
$$

and convex, therefore

$$
\int_{0}^{T} \Phi^{*}\left(t, \Lambda u_{n}(t)\right) d t \leq \int_{0}^{T} \Phi^{*}(t, \Lambda u(t)) d t-\int_{0}^{T}\left\langle\nabla \Phi^{*}\left(t, \Lambda u_{n}(t)\right), \Lambda u(t)-\Lambda u_{n}(t)\right\rangle d t
$$

Taking limits and making use of (3.26) and (3.27), we deduce that

$$
\limsup _{n \rightarrow \infty} \int_{0}^{T} \Phi^{*}\left(t, \Lambda u_{n}(t)\right) d t \leq \int_{0}^{T} \Phi^{*}(t, \Lambda u(t)) d t
$$

Inequalities (3.28) and (3.29) then together imply that

$$
\lim _{n \rightarrow \infty} \int_{0}^{T} \Phi^{*}\left(t, \Lambda u_{n}(t)\right) d t=\int_{0}^{T} \Phi^{*}(t, \Lambda u(t)) d t .
$$

This together with the compact embedding of $W^{2, p^{\prime}}[0, T]$ in $L^{p}[0, T], C[0, T]$ and $C^{1}[0, T]$ implies that

$$
\begin{aligned}
\lim _{n \rightarrow \infty} I\left(u_{n}\right)= & \lim _{n \rightarrow \infty} \int_{0}^{T}\left[\Phi^{*}\left(t, \Lambda u_{n}(t)\right)-\Phi\left(t, u_{n}(t)\right)\right] d t+\Psi_{1}^{*}\left(-\dot{u}_{n}(0)\right)+\Psi_{2}^{*}\left(\dot{u}_{n}(T)\right) \\
& \quad-\Psi_{1}\left(u_{n}(0)\right)-\Psi_{2}\left(u_{n}(T)\right) \\
= & \int_{0}^{T}\left[\Phi^{*}(t, \Lambda u(t))-\Phi(t, u(t))\right] d t+\Psi_{1}^{*}(-\dot{u}(0))+\Psi_{2}^{*}(\dot{u}(T)) \\
& \quad-\Psi_{1}(u(0))-\Psi_{2}(u(T)) \\
= & I(u)
\end{aligned}
$$

from which we obtain $I(u)=c$ and, in particular, $u$ is nontrivial. It also follows from $I^{\prime}\left(u_{n}\right) \rightarrow 0$, together with the fact that $I^{\prime}$ is weakly continuous in $W^{2, p^{\prime}}[0, T]$, that $I^{\prime}(u)=0$. This completes the proof for the case $p=q$.

The proof for the case of $q>p$ relies on a variant of the mountain pass theorem introduced in [8], which we will briefly describe. Let $X$ be a real Banach space and $I$ a function on $X$ of the form $I=F+G$, where $F \in C^{1}(X, \mathbb{R})$ and $G: X \rightarrow(-\infty, \infty]$ is proper, convex and lower semi-continuous. We define critical points of $I$ to be those points $u \in \operatorname{Dom}(G) \subseteq X$ satisfying

$$
\left\langle F^{\prime}(u), v-u\right\rangle_{X^{*} \times X}+G(v)-G(u) \geq 0 \quad \text { for all } v \in X .
$$

$c \in \mathbb{R}$ is called a critical value if $I^{-1}(c)$ contains a critical point. $I$ is said to satisfy the Palais-Smale condition if each sequence $\left\{u_{n}\right\}_{n \in \mathbb{N}}$ in $X$, such that $I\left(u_{n}\right) \rightarrow c \in \mathbb{R}$ and

$$
\left\langle F^{\prime}\left(u_{n}\right), v-u_{n}\right\rangle_{X^{*} \times X}+G(v)-G(u) \geq\left\langle z_{n}, v-u_{n}\right\rangle_{X^{*} \times X} \quad \text { for all } v \in X
$$


where $z_{n} \rightarrow 0$, has a convergent subsequence. Now suppose that $I=F+G$ satisfies the Palais-Smale condition and that the following conditions hold:

(i) $I(0)=0$ and there exist $\delta, \rho>0$ such that $\left.I\right|_{\partial B_{\rho}} \geq \delta$;

(ii) $I(e) \leq 0$ for some $e \notin \bar{B}_{\rho}$.

Then $I$ has a critical value $c \geq \delta$ given by

$$
c=\inf _{\gamma \in \Gamma} \sup _{t \in[0,1]} I(\gamma(t)),
$$

where $\Gamma=\{\gamma \in C([0,1], X): \gamma(0)=0, \gamma(1)=e\}$. In light of this result, the proof for the case of $q>p$ then follows in the same way as the proof for $q=p$.

\section{Further remarks}

Remark 4.1. Assuming that $f(t)=0$, one can think of the functional $I$ in (1.5) as a function of two parameters $p, q^{\prime}>1$ :

$$
I_{p, q^{\prime}}(u)=\frac{1}{q^{\prime}} \int_{0}^{T}|A(t) u(t)-\ddot{u}(t)|^{q^{\prime}} d t-\frac{1}{p} \int_{0}^{T}|u(t)|^{p} d t .
$$

It follows that $I_{p, p^{\prime}}=I$. This functional is continuously differentiable on $W_{\text {per }}^{2, q^{\prime}}[0, T]$. Therefore, because of the compact embedding

$$
W_{\text {per }}^{2, q^{\prime}}[0, T] \hookrightarrow L^{p}[0, T],
$$

for $p>1$ the functional $I_{p, q^{\prime}}$ is lower semi-continuous. It is also straightforward that $I_{p, q^{\prime}}$ satisfies the mountain pass geometry and the condition for $q^{\prime}<p$. Note that this functional is bounded from below and coercive for $q^{\prime}>p$. It then follows that the functional $I_{p, q^{\prime}}$ has a critical point $u \in W_{\text {per }}^{2, q^{\prime}}[0, T]$. If we set

$$
v(t)=|A(t) u(t)-\ddot{u}(t)|^{q^{\prime}-2}(A(t) u(t)-\ddot{u}(t))
$$

then $(u, v)$ is a solution of the Hamiltonian system

$$
\begin{cases}A(t) v(t)-\ddot{v}(t)=|u(t)|^{q-2} u(t), & t \in(0, T), \\ A(t) u(t)-\ddot{u}(t)=|v(t)|^{p-2} v(t), \quad t \in(0, T), & \\ v(0)=v(T), \dot{v}(0)=\dot{v}(T), & \\ u(0)=u(T), \dot{u}(0)=\dot{u}(T) .\end{cases}
$$

Note that the standard variational formulation to prove existence for the above system amounts to finding critical points for the functional

$F(u, v)=\frac{1}{p} \int_{0}^{T}|u(t)|^{p} d t+\frac{1}{q} \int_{0}^{T}|v(t)|^{q} d t-\int_{0}^{T}\langle A(t) u(t), v(t)\rangle d t+\int_{0}^{T}\langle\dot{u}(t), \dot{v}(t)\rangle d t$ on $W^{1,2}[0, T]$. This functional is strongly indefinite and one needs to use linking type theorems to deal with this situation even for the case $q^{\prime}>p$ for which one just needs to minimize the proposed functional (4.1). 
We also note that this new functional turns out to be extremely useful in Hamiltonian systems of partial differential equations. Indeed, in our forthcoming work we shall discuss the existence of solutions for the elliptic system with Neumann boundary condition

$$
\begin{cases}-\Delta v+v=|u|^{q-2} u+\lambda|u|^{r-2} u, & x \in \Omega, \\ -\Delta u+u=|v|^{p-2} v, & x \in \Omega, \\ \frac{\partial u}{\partial n}=\frac{\partial v}{\partial n}=0, & x \in \partial \Omega\end{cases}
$$

where $r<q$ and $p$ and $q$ are on the critical hyperbola

$$
\frac{1}{p}+\frac{1}{q}=\frac{N-2}{N} .
$$

We refer the interested reader to [6] for more details on this problem.

\title{
References
}

[1] J.-P. Aubin and I. Ekeland, 'Second-order evolution equations associated with convex Hamiltonians', Canad. Math. Bull. 23(1) (1980), 81-94.

[2] I. Ekeland, Convexity Methods in Hamiltonian Mechanics (Springer, Berlin, 1990).

[3] I. Ekeland and R. Temam, Convex Analysis and Variational Problems (American Elsevier, New York, 1976).

[4] N. Ghoussoub and A. Moameni, 'Selfdual variational principles for periodic solutions of Hamiltonian and other dynamical systems', Comm. Partial Differential Equations 32(4-6) (2007), 771-795.

[5] N. Ghoussoub and A. Moameni, 'Anti-symmetric Hamiltonians (II): variational resolutions for Navier Stokes and other nonlinear evolutions', Ann. Inst. H. Poincaré Anal. Non Linéaire 26(1) (2009), 223-255.

[6] A. Moameni, 'A variational principle associated with a certain class of boundary value problems', Differential Integral Equations 23(3-4) (2010), 253-264.

[7] A. Moameni, 'Nonconvex self-dual Lagrangians: new variational principles of symmetric boundary value problems', J. Funct. Anal. 260 (2011), 2674-2715.

[8] A. Szulkin, 'Minimax principles for lower semicontinuous functions and applications to nonlinear boundary value problems', Ann. Inst. H. Poincaré Anal. Non Linéaire 3(2) (1986), 77-109.

\author{
MARK LEWIS, Department of Mathematics and Statistics, Queen's University, \\ Kingston, ON, Canada K7L 3N6 \\ e-mail: mlewis@mast.queensu.ca
}

\author{
ABBAS MOAMENI, Department of Mathematical Sciences, \\ Sharif University of Technology, Tehran, Iran \\ e-mail: momeni@mast.queensu.ca
}

